\title{
Identification of a Potential Target of Capsaicin by Computational Target Fishing
}

\author{
Xuan-yi Ye, ${ }^{1}$ Qing-zhi Ling, ${ }^{2}$ and Shao-jun Chen ${ }^{2}$ \\ ${ }^{1}$ College of Ecology, Lishui University, Lishui, Zhejiang 323000, China \\ ${ }^{2}$ Department of Traditional Chinese Medicine, Zhejiang Pharmaceutical College, Ningbo 315100, China \\ Correspondence should be addressed to Shao-jun Chen; chenshaojun@hotmail.com
}

Received 15 September 2015; Revised 17 November 2015; Accepted 18 November 2015

Academic Editor: Ki-Wan Oh

Copyright (c) 2015 Xuan-yi Ye et al. This is an open access article distributed under the Creative Commons Attribution License, which permits unrestricted use, distribution, and reproduction in any medium, provided the original work is properly cited.

\begin{abstract}
Capsaicin, the component responsible for the pungency of chili peppers, shows beneficial effects in many diseases, although the underlying mechanisms remain unclear. In the present study, the potential targets of capsaicin were predicted using PharmMapper and confirmed via chemical-protein interactome (CPI) and molecular docking. Carbonic anhydrase 2 was identified as the main disease-related target, with the pharmacophore model matching well with the molecular features of capsaicin. The relation was confirmed by CPI and molecular docking and supported by previous research showing that capsaicin is a potent inhibitor of carbonic anhydrase isoenzymes. The present study provides a basis for understanding the mechanisms of action of capsaicin or those of other natural compounds.
\end{abstract}

\section{Introduction}

Capsaicin (Figure 1), the component responsible for the pungency of chili peppers, is an alkaloid from the Capsicum species, which is used worldwide in foods, spices, and medicines [1-4]. Capsaicin has been used as traditional medicine to treat muscular pain and headaches, to improve circulation, for its gastrointestinal protective effects, and to fight against many types of cancer [4,5]. It is commonly added to herbal formulations because it acts as a catalyst for other herbs and aids in their absorption [4]. As a result, capsaicin has become an exciting pharmacological agent and its utility in different clinical conditions is being explored [1]. However, the mechanisms underlying the therapeutic effects of capsaicin remain unclear [1].

Target fishing, or target identification, is an important step in modern drug development that explores the mechanism of action of bioactive small molecules by identifying their interacting proteins $[6,7]$. In recent years, a large number of computational target fishing methods have been developed [8]. For example, reverse or inverse docking represents a useful tool that involves docking a small-molecule drug/ligand into the potential binding cavities of a set of clinically relevant macromolecular targets [9]. Identification of the top-ranking targets based on their binding affinity with the drug/ligand may be relevant for drug repositioning and/ or rescue [9]. In recent work from our group, computational tools were used to identify targets of Danshensu and Tanshinone IIA $[10,11]$. Computational target fishing technologies have increased our ability to efficiently and effectively screen for targets in a high-throughput format, which is expected to have a large impact on drug development $[6,8]$.

In the present study, potential targets of capsaicin were predicted by reverse docking and confirmed via chemicalprotein interactome $(\mathrm{CPI})$ and molecular docking. The present study describes a computational drug repositioning method and explores its potential for elucidating the mechanism of action of natural compounds.

\section{Methods}

2.1. Targets Predicted by PharmMapper. PharmMapper is a web server for potential drug target identification based on the use of a pharmacophore mapping approach [12]. It automatically finds the best mapping poses of the query molecule against all the pharmacophore models in PharmTargetDB and lists the top $\mathrm{N}$ best-fitted hits with appropriate target 


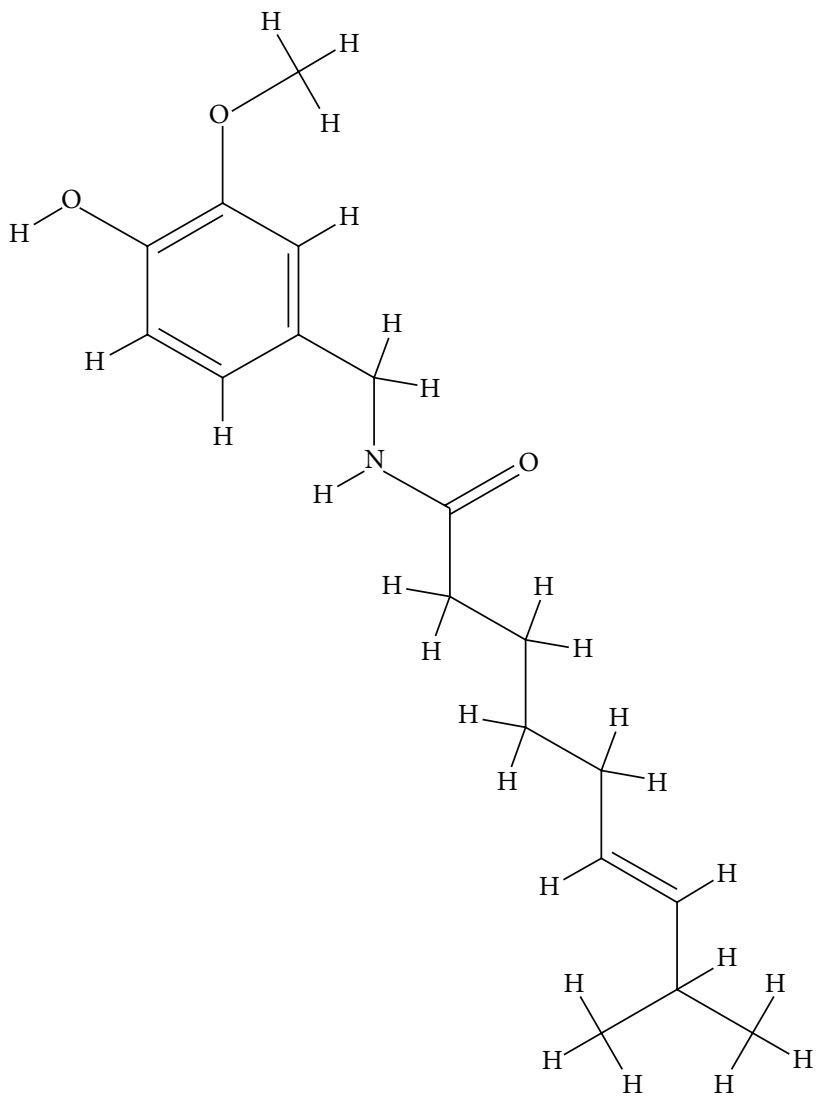

FIGURE 1: The chemical structure of capsaicin (PubChem CID: 1548943).

annotations, as well as the aligned poses of the respective molecules [12].

The molecular file of capsaicin was downloaded from the PubChem database (CID: 1548943) and uploaded to the PharmMapper server. The search started using the maximum generated conformations at 300 by selecting "all targets (7302)" option and default value of 300 for the number of reserved matched targets as described previously [10, 11, 13]. The default settings were used for other parameters.

2.2. Targets Checked by the CPI. The CPI refers to the interaction information of a panel of chemicals across a panel of target proteins in terms of binding strength and binding conformation for each chemical-protein pocket pair [14]. Both DRAR-CPI and DDI-CPI are the servers for computational drug repositioning via the CPI $[15,16]$.

The molecular file of capsaicin was downloaded and pretreated following the web instructions as described previously $[11,15]$. Then, it was submitted to the DRAR-CPI and DDICPI servers. Parameters were set to default values.

2.3. Molecular Docking. Molecular docking is a computational procedure that attempts to predict noncovalent binding of macromolecules or a macromolecule (receptor) and a small molecule (ligand) efficiently [17]. Autodock Vina in PyRx 0.8 is a new program for molecular docking and virtual screening that has been widely used [17-19].
The target protein was prepared using the protein preparing tool in TCM Database@Taiwan (http://dock.cmu.edu .tw/ligand.php), which can extract ligands from binding sites, protonate protein structures, and show ligand coordinates and radius information as described previously [10, 11]. Then, the ligand capsaicin was pretreated through OpenBabel in PyRx 0.8. During the docking procedure, the grid box was centered to cover the binding site residues and to allow the ligand to move freely $[10,11]$. The box was set to $10 \times 10 \times$ $10 \mathrm{~nm}$, and the center coordinates are shown in Table 2. Other parameters were set to default values.

2.4. Visualization. The $3 \mathrm{D}$ visualizations of the complex structure were performed using soft PyMol, and the diagrams of chemical-protein interactions were prepared using Ligplot software.

\section{Results}

3.1. Target Prediction by PharmMapper. Ranking by fit score in descending order and the top ten disease-related targets are shown in Table 1. Carbonic anhydrase 2 (CA2) (PDB ID: $1 \mathrm{BNV}, 1 \mathrm{I} 9 \mathrm{Q}$, and 1I9O) ranked number one, three, and nine respectively. The pharmacophore model (1BNV) shows three hydrophobic sites, one donor, and three acceptors (Figure 2). Moreover, the pharmacophore model showed that CA2 is well matched with capsaicin (Figure 2). These results indicate that 
TABLE 1: Top ten potential disease-related targets of capsaicin predicted by PharmMapper.

\begin{tabular}{lcccc}
\hline Rank & PDB ID & Name & Fit score & Disease \\
\hline 1 & $1 \mathrm{BNV}$ & Carbonic anhydrase 2 & 4.856 & Autosomal recessive osteopetrosis type 3 \\
2 & $1 \mathrm{IZ2}$ & Alpha-1-antitrypsin & 4.727 & Chronic obstructive pulmonary disease \\
3 & $1 \mathrm{I} 9 \mathrm{Q}$ & Carbonic anhydrase 2 & 4.581 & Autosomal recessive osteopetrosis type 3 \\
4 & $5 \mathrm{P} 21$ & GTPase HRas & 4.447 & Costello syndrome, cancer \\
5 & $1 \mathrm{~B} 0 \mathrm{~F}$ & Leukocyte elastase & 4.301 & Cyclic hematopoiesis \\
6 & 2DUX & Aldose reductase & 4.228 & Diabetes, galactosemia \\
7 & 3BYS & Protooncogene tyrosine-protein kinase LCK & 4.225 & Leukemias \\
8 & $1 \mathrm{RLB}$ & Transthyretin & 4.028 & Amyloidosis \\
9 & $1 \mathrm{I} 9 \mathrm{O}$ & Carbonic anhydrase 2 & 4.002 & Autosomal recessive osteopetrosis type 3 \\
10 & $1 \mathrm{R} 1 \mathrm{H}$ & Neprilysin & 3.982 & Acute lymphocytic leukemia \\
\hline
\end{tabular}

TABLE 2: Results of capsaicin-CA interactome by DRAR-CPI and DDI-CPI.

\begin{tabular}{|c|c|c|c|c|c|c|}
\hline \multicolumn{4}{|c|}{ DRAR-CPI } & \multicolumn{3}{|c|}{ DDI-CPI } \\
\hline PDB ID & Name & Docking score & $Z^{\prime}$-score & PDB ID & Name & Docking score \\
\hline 1JD0 & CA 12 & -45.9539 & 1.48099 & $\begin{array}{l}3 \mathrm{CZV} \\
2 \mathrm{FOY}\end{array}$ & $\begin{array}{l}\text { CA } 13 \\
\text { CA } 1\end{array}$ & $\begin{array}{l}-6.4 \\
-6.1\end{array}$ \\
\hline $1 \mathrm{Z93}$ & CA 3 & -41.1238 & 1.79532 & $\begin{array}{l}2 \mathrm{FOU} \\
3 \mathrm{FW} 3\end{array}$ & $\begin{array}{l}\text { CA } 2 \\
\text { CA } 4\end{array}$ & $\begin{array}{l}-5.9 \\
-5.7\end{array}$ \\
\hline
\end{tabular}

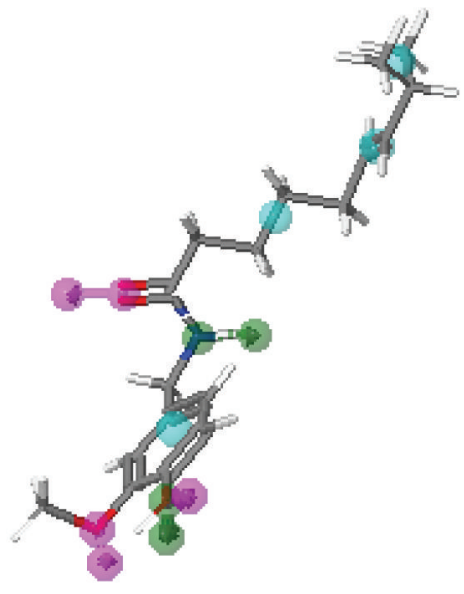

(a)

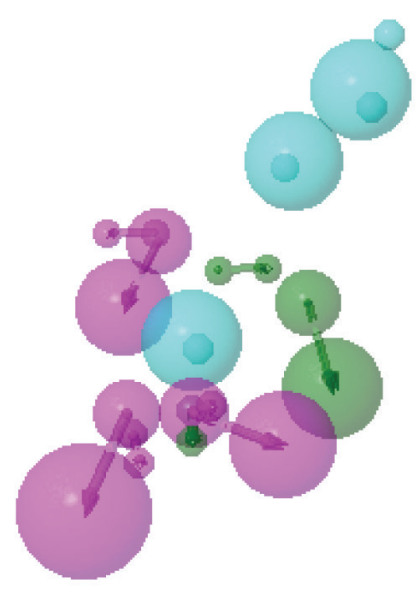

(b)

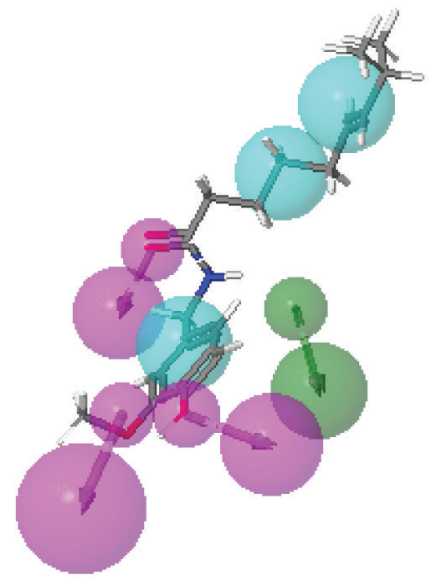

(c)

FIGURE 2: Alignment of capsaicin and pharmacophore model of CA2. (a) Capsaicin features. (b) Pharmacophore model of CA2. (c) Molecular and pharmacophore model. Note: pharmacophore features are indicated by color as follows: hydrophobic, cyan; positive, blue; negative, red; donor, green; and acceptor, magenta.

CA2 may be a potential target of capsaicin. Therefore, CA2 was selected for further investigation.

3.2. Targets Verified by Chemical-Protein Interactome. When a drug is uploaded to the DRAR-CPI server, it is "hybridized" with all targets using the DOCK program [15]. Table 2 shows the results of DRAR-CPI for capsaicin-CA (12 and 3), including the docking score and $Z^{\prime}$-score.

When a molecule is submitted to DDI-CPI, the server will dock it across 611 human proteins, generating a CPI profile that can be used as a feature vector of the preconstructed prediction model [16]. As shown in Table 2, four CA isoforms, including CA1, 2, 4, and 13, docked with capsaicin. The docking score of capsaicin-CA2 was $-5.9 \mathrm{kcal} / \mathrm{mol}$. Furthermore, the binding pattern of capsaicin-CA2 complex can be visualized in Figure 3.

3.3. Molecular Docking. Upon docking using Autodock Vina in PyRx 0.8, the lowest binding energy of the capsaicin-CA2 complex was $-6.2 \mathrm{kcal} / \mathrm{mol}$ (Table 3 ). As shown in Figure 4, the ligand capsaicin formed four hydrogen bonds with the active site residues (Gln92, Thr199, and Thr200). A number 


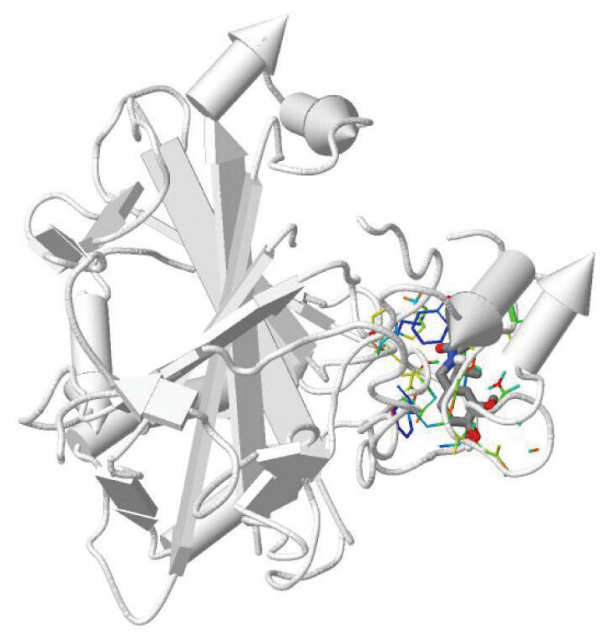

FIGURE 3: Visualization of a capsaicin-CA2 complex captured from the DDI-CPI server. Note: protein chain: rocket; drug: stick; key residues: colorful line.

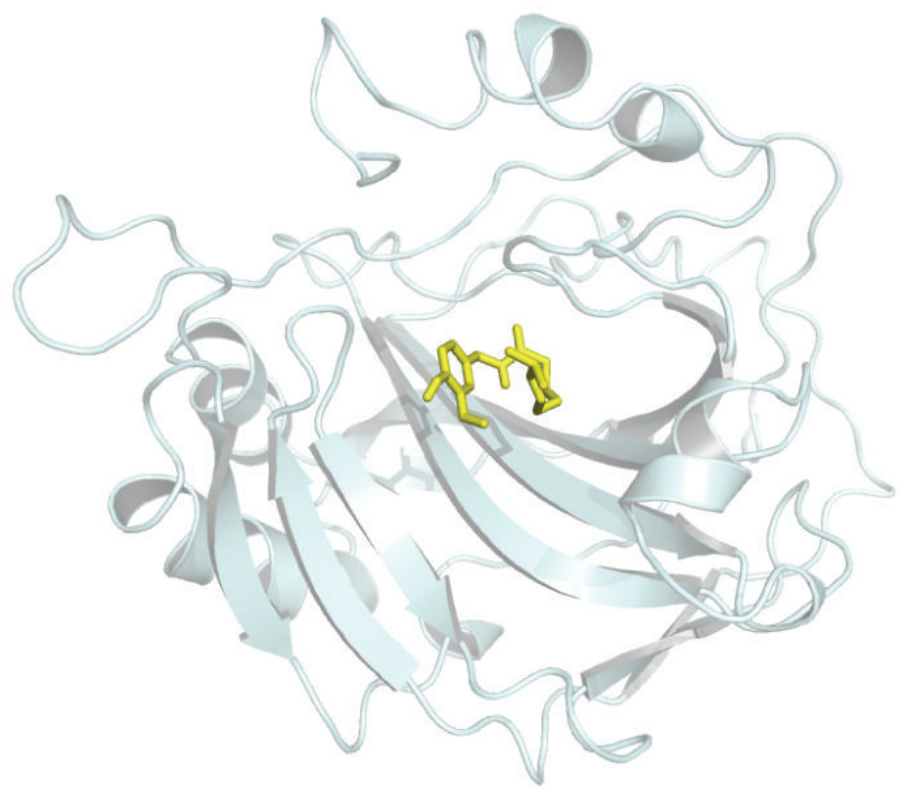

(a)

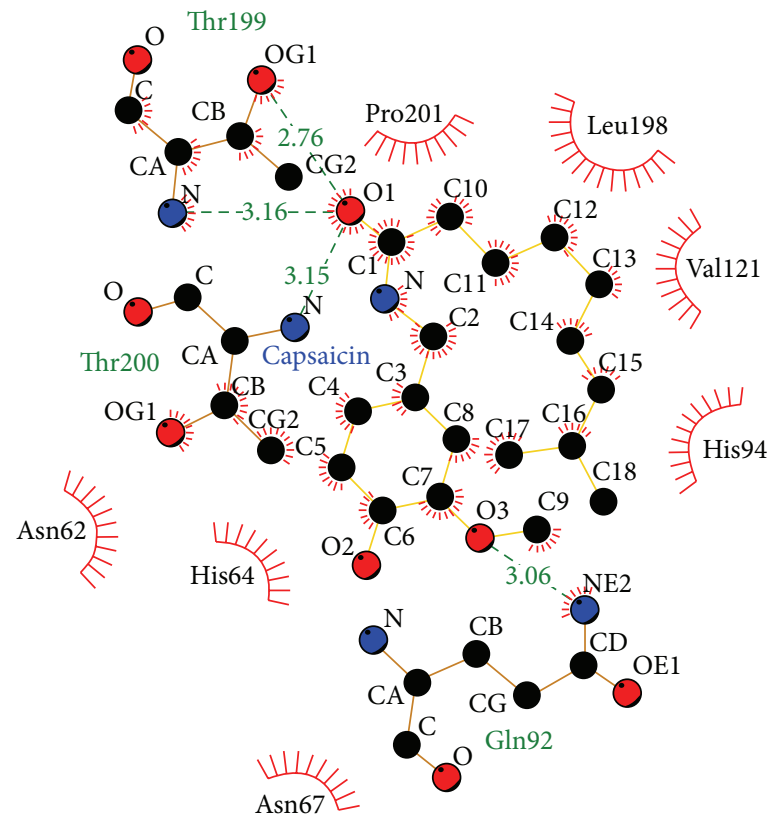

(b)

Figure 4: Molecular interactions between capsaicin and CA2. (a) 3D structure of the CA2 (1BVN)-capsaicin complex by PyMol. Capsaicin: yellow; hydrogen bond: red dash line. (b) 2D interaction scheme by Ligplot. Capsaicin: yellow; C, N, and O atoms are represented in black, blue, and red; hydrophobic contacts are presented in brick red.

TABLE 3: The center coordinates of the binding site and the lowest binding energy by molecular docking.

\begin{tabular}{lccc}
\hline PDB ID & Name & Center $(x \times y \times z)$ & $\begin{array}{c}\text { Binding } \\
\text { affinity }\end{array}$ \\
\hline 1BNV & $\begin{array}{c}\text { Carbonic } \\
\text { anhydrase 2 }\end{array}$ & $-4.03 \times 4.83 \times 14.43$ & $-6.2 \mathrm{kcal} / \mathrm{mol}$ \\
\hline
\end{tabular}

of hydrophobic interactions are depicted in Figure 4(b). Many residues, including Asn62, His64, Asn67, His94, Val121,
Leu198, and Pro201, formed hydrophobic contacts with capsaicin.

\section{Discussion}

The identification of drug targets in the human genome is important for the development of new pharmaceutical products and the allocation of resources in academic and industrial biomedical research [20]. Various innovative computational tools have been developed to integrate biological data such as regulatory networks, molecular pathways, and 
cell phenotypes, which facilitates the interpretation and prediction of the biological activities of drugs and their targets $[8,21]$. Reverse or inverse docking is a powerful tool for drug repositioning and drug rescue [9]. Recently, PharmMapper, a reverse docking server, was used to identify potential targets of small molecules derived from Indigofera species [22] and for the computational prediction of breast cancer targets for 6-methyl-1,3,8-trichlorodibenzofuran [13]. In our previous reports, we used the PharmMapper server to identify potential targets of active compounds from Danshen, a traditional Chinese medicine $[10,11]$. We therefore used PharmMapper, a powerful computational tool, to identify CA2 as the main disease-related target of capsaicin in the present study (Table 1). The CA2 pharmacophore confirmed the alignment of molecular features with capsaicin (Figure 2).

The use of CPI together with systems biology-based integrative computational strategies is an essential complement, if not an alternative, to current drug evaluation methods [14]. In a DRAR-CPI job, potential drug targets with $Z^{\prime}$-score $<-1$ are considered as the favorable targets and those with $Z^{\prime}$-score $>1$ are considered as the unfavorable targets [15]. In a DDI-CPI job, the docking scores for each drug in the training set are generated against the 611 library targets [16]. In the present study, $Z^{\prime}$-score in DRAR-CPI and docking score in DDICPI indicated that CA2 is a target of capsaicin and should be further investigated. These results were consistent with the reverse docking results (Table 1 ).

Understanding the interactions between proteins and biologically relevant ligands is an important step towards identifying the functions of proteins [23]. The hydrophobic surface of the active site cavity of CA2 contains the residues Ala121 and 135; Val207; Phe91; Leu131, 138, 146, and 109; and Pro201 and 202; and the hydrophilic surface consists of His64, 67, and 200; Asn69; Gln92; Thr199; Tyr7; and Val62 [24]. Thr199 plays a significant role by forming two hydrogen bonds with the carboxyl group of Glu106 and zinc hydroxide [24]. Residues Asn67 and Leu198 protrude towards the $\mathrm{Zn}^{2+}$ ion and reduce the volume of the active site cavity considerably [24]. His64, Asn67, and Gln92 residues are involved in histidine recognition [24]. In short, these residues play key roles in ligand-protein interactions. The original ligand sulfonamide forms hydrogen bonds with residues Gln92, His119, Thr199, and Thr200 and forms hydrophobic interactions with Phe131 [25]. Figure 4(b) shows that capsaicin can form hydrogen bonds with Gln92, Thr199, and Thr200 and has hydrophobic interactions with Asn62, His64, Asn67, His94, Val121, Leu198, and Pro201. The structural details indicate that capsaicin may interact with CA2 via these key residues [24]. A previous study reported that capsaicin has $K_{i}$ of $696.15 \mu \mathrm{M}$ against hCA I and of $208.37 \mu \mathrm{M}$ against hCA II, showing unique inhibition profiles against both CA isoforms I and II and suggesting that capsaicin is a selective inhibitor of both cytosolic CA isoenzymes [26].

CAs, a group of ubiquitously expressed metalloenzymes, are involved in numerous physiological and pathological processes, including gluconeogenesis, lipogenesis, ureagenesis, tumorigenicity, and the growth and virulence of various pathogens [27]. In addition to the established role of CA inhibitors (CAIs) as diuretics and antiglaucoma drugs, the potential of
CAIs as novel antiobesity, anticancer, anti-infective, and antiAlzheimer's drugs was recently shown [27]. Taken together with previous results, our findings suggest that capsaicin may play a role in these diseases through its effect on CA2.

In the present study, potential targets of capsaicin were identified using PharmMapper and confirmed via CPI and Autodock Vina. Our results identified CA2 as a potential target of capsaicin, although further studies are necessary to determine their precise interaction. The present study demonstrated that computational drug repositioning is a useful strategy to screen for targets of capsaicin or other natural compounds and suggested a mechanism of action of capsaicin.

\section{Conflict of Interests}

The authors declare that there is no conflict of interests regarding the publication of this paper.

\section{Acknowledgments}

This work was supported by Zhejiang Provincial Natural Science Foundation of China (LY15H280009), Ningbo Municipal Natural Science Foundation (2015A610280), and the Administration of Traditional Chinese Medicine of Zhejiang Province (2014ZB110).

\section{References}

[1] S. K. Sharma, A. S. Vij, and M. Sharma, "Mechanisms and clinical uses of capsaicin," European Journal of Pharmacology, vol. 720, no. 1-3, pp. 55-62, 2013.

[2] M. De Lourdes Reyes-Escogido, E. G. Gonzalez-Mondragon, and E. Vazquez-Tzompantzi, "Chemical and pharmacological aspects of capsaicin," Molecules, vol. 16, no. 2, pp. 1253-1270, 2011.

[3] J. Szolcsányi, "Forty years in capsaicin research for sensory pharmacology and physiology," Neuropeptides, vol. 38, no. 6, pp. 377-384, 2004.

[4] X.-F. Huang, J.-Y. Xue, A.-Q. Jiang, and H.-L. Zhu, "Capsaicin and its analogues: structure-activity relationship study," Current Medicinal Chemistry, vol. 20, no. 21, pp. 2661-2672, 2013.

[5] I. Díaz-Laviada and N. Rodríguez-Henche, "The potential antitumor effects of capsaicin," Progress in Drug Research, vol. 68, pp. 181-208, 2014.

[6] L. Wang and X.-Q. Xie, "Computational target fishing: what should chemogenomics researchers expect for the future of in silico drug design and discovery?" Future Medicinal Chemistry, vol. 6, no. 3, pp. 247-249, 2014.

[7] B. Lomenick, R. W. Olsen, and J. Huang, "Identification of direct protein targets of small molecules," ACS Chemical Biology, vol. 6, no. 1, pp. 34-46, 2011.

[8] A. Cereto-Massagué, M. J. Ojeda, C. Valls, M. Mulero, G. Pujadas, and S. Garcia-Vallve, "Tools for in silico target fishing," Methods, vol. 71, pp. 98-103, 2015.

[9] P. S. Kharkar, S. Warrier, and R. S. Gaud, "Reverse docking: a powerful tool for drug repositioning and drug rescue," Future Medicinal Chemistry, vol. 6, no. 3, pp. 333-342, 2014. 
[10] S.-J. Chen and J.-L. Ren, "Identification of a potential anticancer target of Danshensu by inverse docking," Asian Pacific Journal of Cancer Prevention, vol. 15, no. 1, pp. 111-116, 2014.

[11] S.-J. Chen, "A potential target of Tanshinone IIA for acute promyelocytic leukemia revealed by inverse docking and drug repurposing," Asian Pacific Journal of Cancer Prevention, vol. 15, no. 10, pp. 4301-4305, 2014.

[12] X. Liu, S. Ouyang, B. Yu et al., "PharmMapper server: a web server for potential drug target identification using pharmacophore mapping approach," Nucleic Acids Research, vol. 38, no. 2, pp. W609-W614, 2010.

[13] K. N. Chitrala and S. Yeguvapalli, "Computational prediction and analysis of breast cancer targets for 6-methyl-1, 3, 8trichlorodibenzofuran," PLOS ONE, vol. 9, no. 11, Article ID e109185, 2014.

[14] L. Yang, K. J. Wang, L. S. Wang et al., "Chemical-protein interactome and its application in off-target identification," Interdisciplinary Sciences: Computational Life Sciences, vol. 3, no. 1, pp. 22-30, 2011.

[15] H. Luo, J. Chen, L. Shi et al., "DRAR-CPI: a server for identifying drug repositioning potential and adverse drug reactions via the chemical-protein interactome," Nucleic Acids Research, vol. 39, supplement 2, pp. W492-W498, 2011.

[16] H. Luo, P. Zhang, H. Huang et al., "DDI-CPI, a server that predicts drug-drug interactions through implementing the chemical-protein interactome," Nucleic Acids Research, vol. 42, no. 1, pp. W46-W52, 2014.

[17] O. Trott and A. J. Olson, "AutoDock Vina: improving the speed and accuracy of docking with a new scoring function, efficient optimization, and multithreading," Journal of Computational Chemistry, vol. 31, no. 2, pp. 455-461, 2010.

[18] A. Sridhar, S. Saremy, and B. Bhattacharjee, "Elucidation of molecular targets of bioactive principles of black cumin relevant to its anti-tumour functionality-an Insilico target fishing approach," Bioinformation, vol. 10, no. 11, pp. 684-688, 2014.

[19] S. Kumar, L. Jena, K. Mohod, S. Daf, and A. K. Varma, "Virtual screening for potential inhibitors of high-risk human papillomavirus 16 E6 protein," Interdisciplinary Sciences: Computational Life Sciences, vol. 7, no. 2, pp. 136-142, 2015.

[20] M. Rask-Andersen, M. S. Almén, and H. B. Schiöth, "Trends in the exploitation of novel drug targets," Nature Reviews Drug Discovery, vol. 10, no. 8, pp. 579-590, 2011.

[21] J. N. Y. Chan, C. Nislow, and A. Emili, "Recent advances and method development for drug target identification," Trends in Pharmacological Sciences, vol. 31, no. 2, pp. 82-88, 2010.

[22] S. K. Paramashivam, K. Elayaperumal, B. B. Natarajan, M. Ramamoorthy, S. Balasubramanian, and K. Dhiraviam, "In silico pharmacokinetic and molecular docking studies of small molecules derived from Indigofera aspalathoides Vahl targeting receptor tyrosine kinases," Bioinformation, vol. 11, no. 2, pp. 7384, 2015.

[23] J. Yang, A. Roy, and Y. Zhang, "BioLiP: a semi-manually curated database for biologically relevant ligand-protein interactions," Nucleic Acids Research, vol. 41, no. 1, pp. D1096-D1103, 2013.

[24] M. Imtaiyaz Hassan, B. Shajee, A. Waheed, F. Ahmad, and W. S. Sly, "Structure, function and applications of carbonic anhydrase isozymes," Bioorganic and Medicinal Chemistry, vol. 21, no. 6, pp. 1570-1582, 2013.

[25] P. A. Boriack-Sjodin, S. Zeitlin, H.-H. Chen et al., "Structural analysis of inhibitor binding to human carbonic anhydrase II," Protein Science, vol. 7, no. 12, pp. 2483-2489, 1998.
[26] B. Arabaci, I. Gulcin, and S. Alwasel, "Capsaicin: a potent inhibitor of carbonic anhydrase isoenzymes," Molecules, vol. 19, no. 7, pp. 10103-10114, 2014.

[27] C. T. Supuran, "Carbonic anhydrases: novel therapeutic applications for inhibitors and activators," Nature Reviews Drug Discovery, vol. 7, no. 2, pp. 168-181, 2008. 


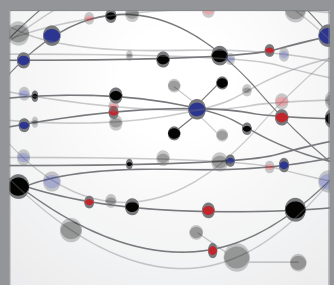

The Scientific World Journal
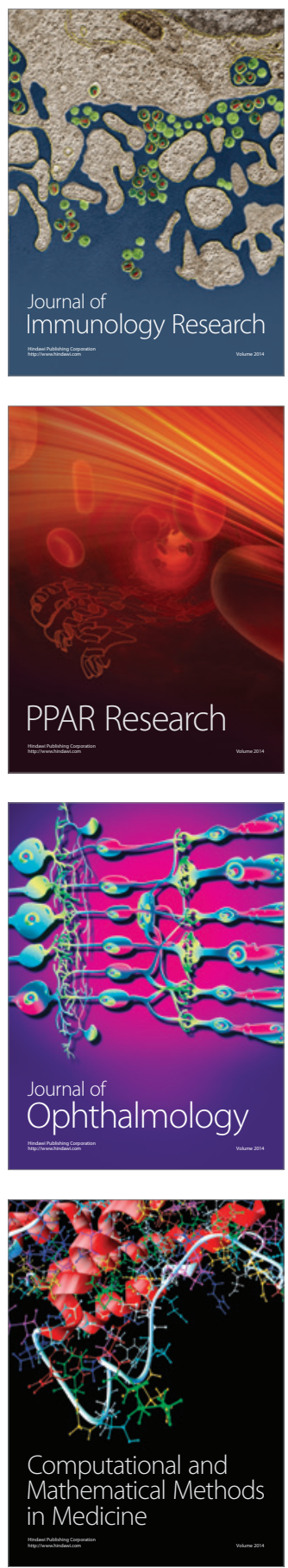

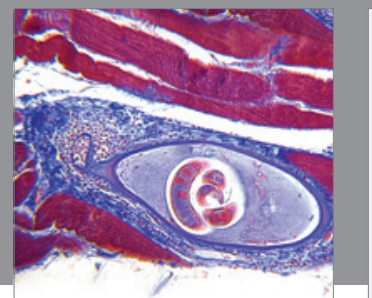

Gastroenterology

Research and Practice
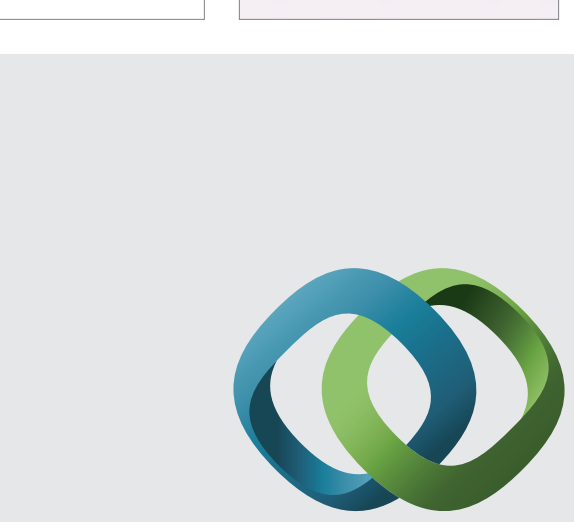

\section{Hindawi}

Submit your manuscripts at

http://www.hindawi.com
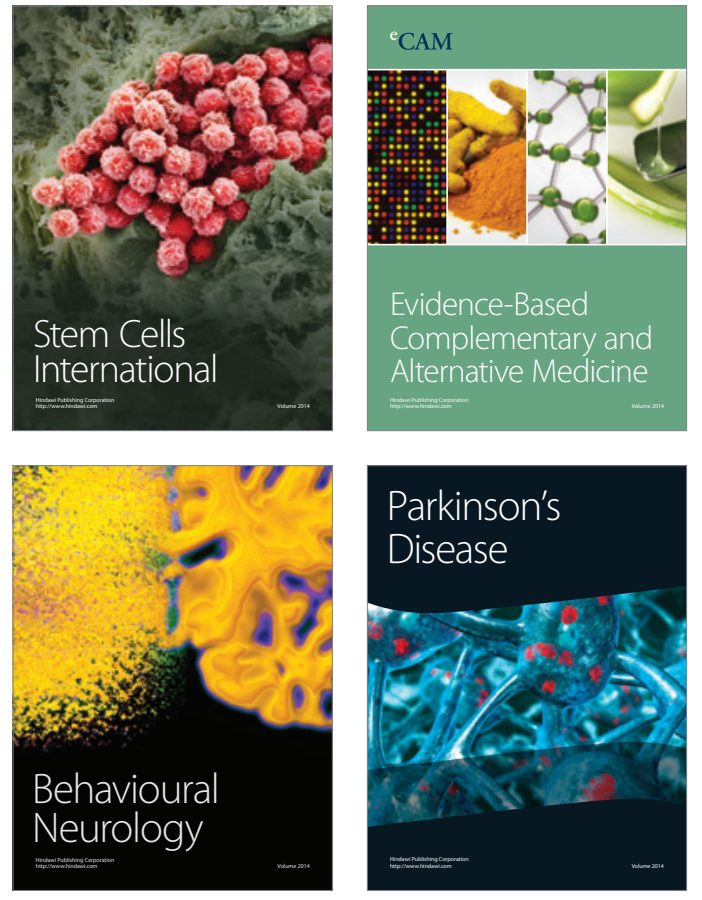
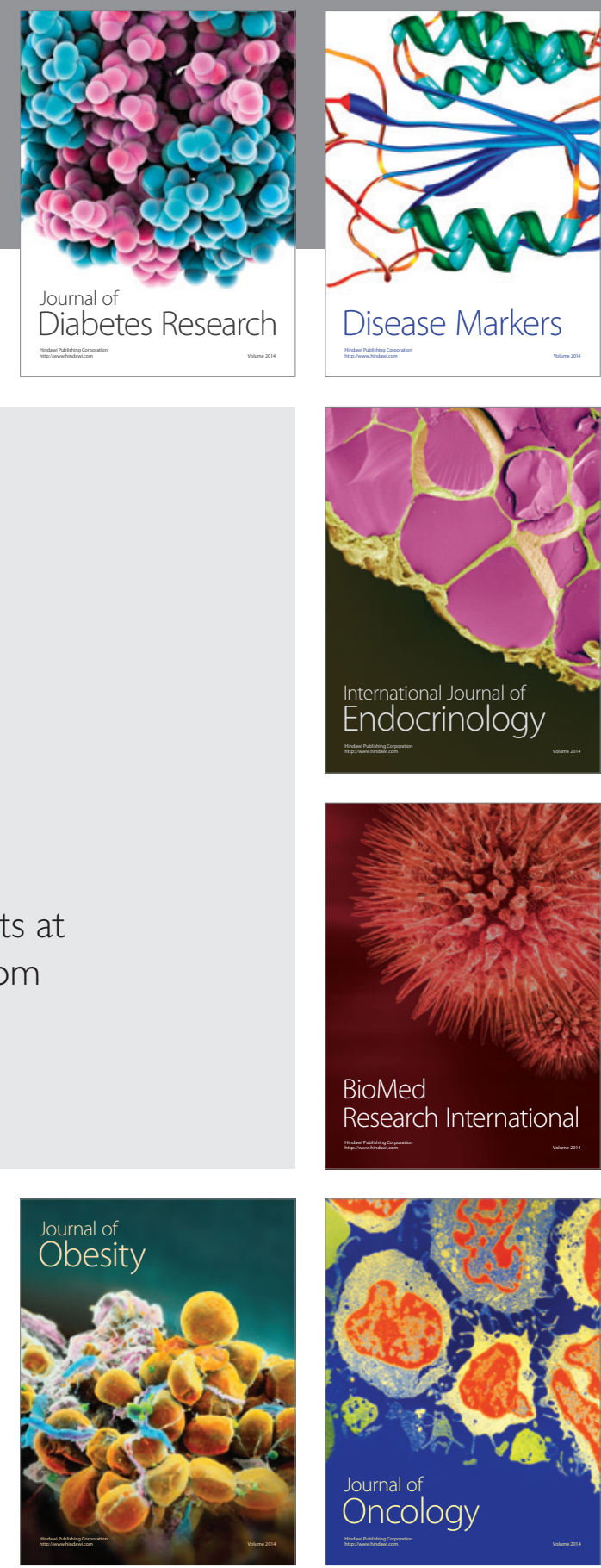

Disease Markers
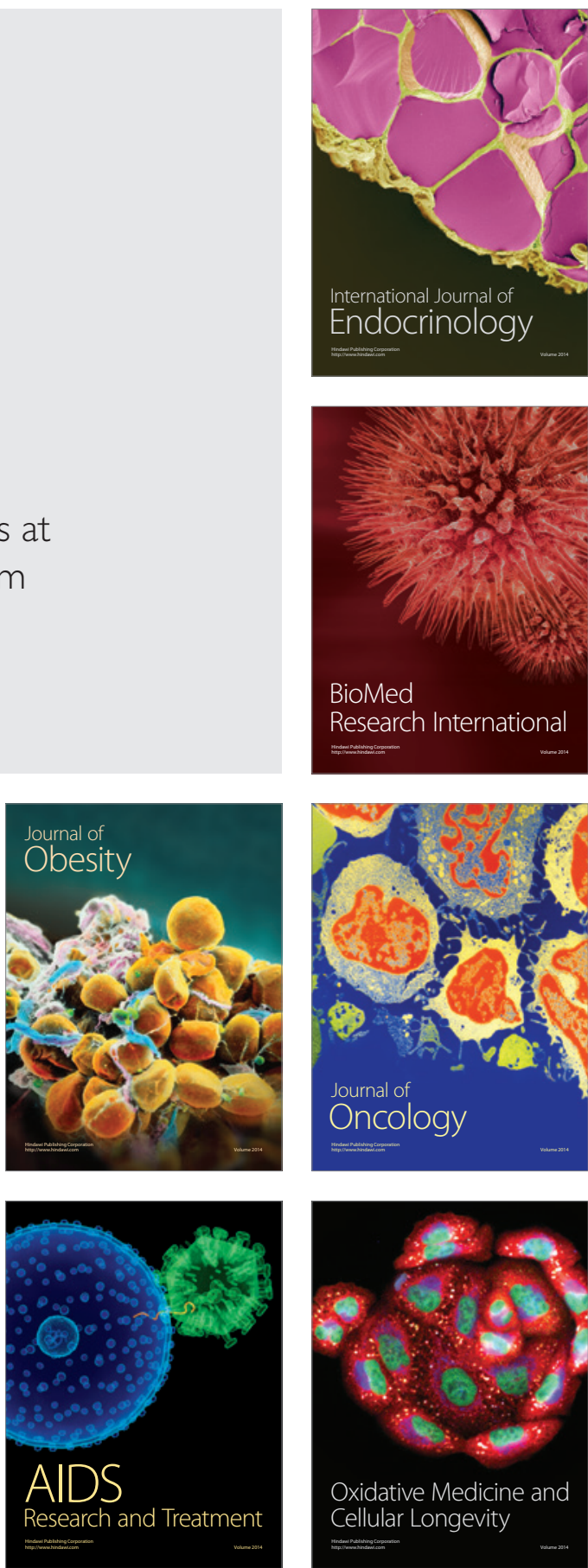